\title{
Transcription factors related to chondrogenesis in pleomorphic adenoma of the salivary gland: a mechanism of mesenchymal tissue formation
}

\author{
Yuka Matsumoto ${ }^{1,2,6}$, Sunao Sato', Takashi Maeda ${ }^{3}$, Mitsunobu Kishino', Satoru Toyosawa', Yu Usami ${ }^{4}$, So-ichi Iwai ${ }^{2}$ \\ Mitsuhiro Nakazawa², Yoshiaki Yura² and Yuzo Ogawa ${ }^{1,5}$
}

In salivary gland pleomorphic adenoma, expression of extracellular matrix (ECM) substances indicates that tumor epithelial cells are becoming chondrogenic and will produce cartilage-like mesenchymal tissues. Sox9, the master transcription factor of chondrogenesis, is expressed in mouse salivary gland cells. To clarify the mechanism behind chondrogenesis in tumor epithelial cells, we examined the expression of transcription factors related to chondrogenesis in tumors and salivary glands. Reverse transcriptase-polymerase chain reaction (RT-PCR), quantitative real-time RT-PCR, and immunostaining were performed on pleomorphic adenoma tissues, salivary gland tissues, and human submandibular gland (HSG) cells. The mRNAs of essential transcription factors for chondrogenesis-Sox9, Sox6, and Sox5-were detected in both tumor and salivary gland tissues. The mRNAs of aggrecan and type II collagen-cartilage-specific ECM substances-were detected only in tumors. Sox 9 and Sox 6 proteins were colocalized in many epithelial cells in tumors and salivary glands. Tumor epithelial cells also possessed aggrecan protein and occasionally type II collagen protein. Moreover, mRNAs for transcription repressors of chondrogenesis $\delta E F 1$ and $A P-2 a$ were detected in both tumors and salivary glands, whereas Twist1 mRNA was detected only in salivary glands and was at significantly low-to-undetectable levels in tumors. Twist 1 protein was localized in the Sox9-expressing salivary gland cells. HSG cells expressed Sox9, Sox6, and Twist1, but not aggrecan or type II collagen, and thus were similar to salivary gland cells. Twist1 depletion by Twist 1 siRNA led to the upregulation of aggrecan and type II collagen mRNA expression in HSG cells. In contrast, forced expression of Twist1, using Twist 1 cDNA, resulted in the downregulation of both these genes. Taken together, these results indicate that salivary gland cells have a potential for chondrogenesis, and Twist1 depletion concomitant with neoplastic transformation, which would permit tumor epithelial cells to produce cartilage-like mesenchymal tissues in salivary gland pleomorphic adenoma. Laboratory Investigation (2016) 96, 16-24; doi:10.1038/labinvest.2015.124; published online 26 October 2015

Pleomorphic adenoma is the most common tumor of the salivary gland. It is characterized by mixed appearance of epithelial and mesenchymal components. ${ }^{1,2}$ A number of ultrastructural and immunohistochemical studies have shown that the mesenchymal component (typically composed of myxoid and chondroid tissues) is epithelial in origin (e.g., Dardick et al. ${ }^{3,4}$ and Erlandson et al. ${ }^{5}$ ). Immunohistochemical and in situ hybridization studies have shown the production of aggrecan and type II collagen, extracellular matrix (ECM) substances specific to cartilage, in the myxoid and chondroid tissues. $^{6-9}$ As aggrecan is also produced in the epithelial component, ${ }^{6,9}$ epithelial cells of pleomorphic adenoma could conceivably become chondrogenic and produce mesenchymal tissues similar to cartilage.

When forced to express SRY-related HMG-box (Sox) genes -that is, SOX9, SOX6, and SOX5-nonchondrogenic cells, including epithelial cells, become chondrogenic and produce type II collagen, aggrecan, and other cartilage markers. ${ }^{10}$ Sox genes encode transcription factors, which control the synthesis of mRNA from specific genes. Sox9, Sox6, and Sox5, the products of SOX9, SOX6, and SOX5, respectively, are essential for chondrogenesis. SOX9, which, when mutated,

\footnotetext{
'Department of Oral Pathology, Osaka University Graduate School of Dentistry, Osaka, Japan; ${ }^{2}$ Department of Oral and Maxillofacial Surgery II, Osaka University Graduate School of Dentistry, Osaka, Japan; ${ }^{3}$ Department of Anatomy and Cell Biology, Osaka University Graduate School of Dentistry, Osaka, Japan; ${ }^{4}$ Clinical Laboratory, Osaka University Dental Hospital, Osaka, Japan and ${ }^{5}$ Department of Oral Health Sciences, Faculty of Nursing and Health Care, Baika Women's University, Osaka, Japan Correspondence: Professor Y Ogawa, DDS, PhD, Department of Oral Health Sciences, Faculty of Nursing and Health Care, Baika Women's University, 2-19-5 Shukunosho, Ibaraki, Osaka 567-8578, Japan.

E-mail: y-ogawa@baika.ac.jp

${ }^{6}$ Current address: Department of Oral and Maxillofacial Surgery, Meiwa Hospital, Hyogo, Japan

Received 3 February 2015; revised 31 August 2015; accepted 14 September 2015
} 
was initially identified as the causative gene of campomelic dysplasia, a severe form of chondrodysplasia in humans, is required for the commitment and differentiation of pluripotent mesenchymal cells toward chondrogenic lineages. ${ }^{11}$ Sox9 is expressed in chondrogenic mesenchymal cells, prechondrocytes, and chondrocytes, with the exception of hypertrophic chondrocytes. In chimeric mice, Sox9-deficient $\left(S o x 9^{-1-}\right)$ cells were excluded from all cartilages, but were present as a juxtaposed mesenchyme that did not express type II collagen, aggrecan, and other chondrocyte marker genes. ${ }^{12}$ Conditional inactivation of Sox9 in limb buds before mesenchymal condensation (before commitment) led to complete absence of chondrocytes, whereas conditional inactivation of Sox9 after mesenchymal condensation resulted in severe generalized chondrodysplasia. ${ }^{13}$

Sox9 in itself is not sufficient for cartilage formation. It requires Sox 5 or Sox 6 to drive overt chondrogenesis. ${ }^{11}$ Sox 5 and Sox6 are very similar in structure and functionally redundant in chondrogenesis. ${ }^{14}$ They are expressed with Sox 9 in prechondrocytes and enhance transcriptional activity of Sox9. Sox $5^{-1-}$ and Sox $6^{-1-}$ individual mutant mice are born

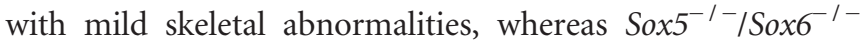
double mutants die in utero with rudimentary and poorly developed cartilage anlagen that express ECM genes, including type II collagen and aggrecan, at low-to-undetectable levels. ${ }^{15}$ In vitro, Sox 5 and Sox6 secure the binding of Sox 9 to chondrocyte-specific enhancer elements in type II collagen, aggrecan, and other chondrocyte genes. ${ }^{16}$

Chondrogenesis is positively regulated by Sox proteins, but negatively regulated by other transcription factors, which inhibit the expression of ECM proteins in chondrogenic cells. Thus, gene expression in chondrogenic cells depends on a balance of positive and negative regulators. ${ }^{17}$ Negative regulators include $\delta E F 1, A P-2 \alpha$, Snail, Slug, Twist1, and C/EBP $\beta .{ }^{18-22}$ Snail, Slug, and C/EBP $\beta$ are expressed by hypertrophic chondrocytes, which indicates that they control terminal chondrocyte differentiation and promote endochondral ossification; $;^{11,19,22} \delta \mathrm{EF} 1, \mathrm{AP}-2 \alpha$, and Twist1 are expressed by chondroprogenitors, which indicates that they control early-phase chondrogenesis. ${ }^{18,20,21,23}$

The aim of our study was to clarify the mechanisms behind chondrogenesis in pleomorphic adenoma epithelial cells. Recently, mouse salivary gland cells, the cells of origin of pleomorphic adenoma, were found to express Sox9. ${ }^{24}$ However, expression of Sox proteins in human salivary gland and pleomorphic adenoma is unknown as far as we know. In the present study, we investigated the expression of positive and negative transcriptional regulators of chondrogenesis in pleomorphic adenoma and salivary gland tissues, and cell lines. Comparison of normal and tumor tissues revealed that in pleomorphic adenoma, Sox 9 and Sox6 expression was sustained, but Twist1 expression was markedly reduced. Further in vitro gain- and loss-of-function experiments demonstrated that knockdown of Twistl in human submandibular gland (HSG) cells increased expression of chondrogenic marker genes aggrecan and type II collagen, whereas the opposite effect was observed when Twistl was overexpressed. Our data suggest that the loss of Twist1 expression has an important role in the formation of cartilage-like tissue in salivary gland pleomorphic adenoma.

\section{MATERIALS AND METHODS \\ Tissues and Cells}

Ethical approval for the use of human materials was obtained from the Ethics Committee of Osaka University Dental Hospital and Graduate School of Dentistry. Formalin-fixed, paraffin-embedded tissue blocks of 15 pleomorphic adenomas and 8 normal salivary glands were selected from the pathology files of Osaka University Dental Hospital, from patients treated during 2011-2013. Pleomorphic adenomas occurred in six major and nine minor salivary glands. The salivary glands were four major and four minor glands. Fresh tissue samples were obtained from four tumors (two in major glands and two in minor glands) and three salivary glands (one major gland and two minor glands), frozen in liquid nitrogen, and stored at $-80^{\circ} \mathrm{C}$ until use.

HSG cells (HSGc in Shirasuna et al..$^{25}$ ), a human submandibular gland cell line, were a kind gift from Prof. Mitsunobu Sato. ${ }^{26}$ They were grown in Dulbecco's modified Eagle's medium supplemented with $10 \%$ fetal bovine serum, penicillin $\mathrm{G}(100 \mathrm{U} / \mathrm{ml})$, and streptomycin $(100 \mu \mathrm{g} / \mathrm{ml})$ at $37^{\circ} \mathrm{C}$ in a humidified $5 \% \mathrm{CO}_{2}$ incubator. Cells between passages 3 and 10 were used in this study.

\section{RNA Isolation and Reverse Transcriptase-Polymerase Chain Reaction}

Total RNA was extracted from fresh tissues and HSG cells with Trizol (Invitrogen, Carlsbad, CA, USA). First-strand cDNA was then made using ReverTra Ace qPCR Master Mix with gDNA Remover (Toyobo, Osaka, Japan). PCR was performed with HotStarTaq DNA Polymerase (Qiagen, Hilden, Germany). Reactions were performed using a DNA Engine PTC-200 (Bio-Rad, Hercules, CA, USA) as follows: $95^{\circ} \mathrm{C}$ for $15 \mathrm{~min}$, followed by 35 cycles ( 40 cycles for type II collagen) of denaturation at $94^{\circ} \mathrm{C}$ for $30 \mathrm{~s}$, annealing for $1 \mathrm{~min}$, and extension at $72{ }^{\circ} \mathrm{C}$ for $1 \mathrm{~min}$. PCR products were separated by $1 \%$ agarose gel electrophoresis. Primer sequences and annealing temperatures are listed in Table 1.

\section{Quantitative Real-Time RT-PCR}

Real-time PCR was performed with Kapa SYBR Fast qPCR Kit (Kapa Biosystems, Boston, MA, USA). Reactions were performed at $95^{\circ} \mathrm{C}$ for $2 \mathrm{~min}$ and then at $95^{\circ} \mathrm{C}$ for $3 \mathrm{~s}$ and $60^{\circ} \mathrm{C}$ for $23 \mathrm{~s}$ for 40 cycles using a Mini Opticon Real-Time PCR system (Bio-Rad). Relative gene expression quantification was determined by the comparative $\Delta C_{\mathrm{t}}$ method and relative expression was normalized to ribosomal protein L13a (RPL13A). Primer sequences are in Table 1. 


\section{Immunostaining}

Sections (5-6 $\mu \mathrm{m}$ thick) were made from paraffin-embedded tissue blocks, mounted on silane-coated glass slides, and used for immunoperoxidase and immunofluorescence staining.

Table 1 Primers used for RT-PCR and qRT-PCR

\begin{tabular}{|c|c|c|c|}
\hline Gene & Primer $\left(5^{\prime} \rightarrow 3^{\prime}\right)$ & $T_{\mathrm{A}}\left({ }^{\circ} \mathrm{C}\right)$ & Size (bp) \\
\hline \multicolumn{4}{|l|}{$R T-P C R$} \\
\hline \multirow[t]{2}{*}{ Sox9 } & F: GATGGCCGAGATGATCCTAA & 59 & 02 \\
\hline & R: CAAGTGGGTAATGCGCTTG & & \\
\hline \multirow[t]{2}{*}{ Sox6 } & F: GCAATTCAAGGGCCACAC & 59 & 130 \\
\hline & R: CTGGACTGACATGCTGGCT & & \\
\hline \multirow[t]{2}{*}{ Sox5 } & F: AGCCAGAGTTAGCACAATAGG & 56 & 619 \\
\hline & R: ATCGGAACACGTTCACACAA & & \\
\hline \multirow[t]{2}{*}{ Aggrecan } & F: CTGCAACTGAAGTGCCC & 56 & 186 \\
\hline & R: CAAGTGGGTAATGCGCTTG & & \\
\hline \multirow[t]{2}{*}{ Type II collagen } & F: CAGGTGAACCTGGACGAGAG & 59 & 86 \\
\hline & R: ACCACGATCTCCCTTGACTC & & \\
\hline \multirow[t]{2}{*}{$\delta E F 1$} & F: GCCAATAAGCAAACGATTCTG & 59 & 101 \\
\hline & R: TTTGGCTGGATCACTITCAAG & & \\
\hline \multirow[t]{2}{*}{$A P-2 a$} & F: GCCCCGTGTCCCTGTCCAA & 60 & 132 \\
\hline & R: TGAGGAGCGAGAGGCGACC & & \\
\hline \multirow[t]{2}{*}{ Twist1 } & F: GGAGTCCGCAGTCTTACGAG & 59 & 201 \\
\hline & R: TCTGGAGGACCTGGTAGAGG & & \\
\hline \multirow[t]{2}{*}{ GAPDH } & F: CCATCACCATCTTCCAGGAG & 60 & 322 \\
\hline & R: GCATGGACTGTGGTCATGA & & \\
\hline \multicolumn{4}{|l|}{$q R T-P C R$} \\
\hline \multirow[t]{2}{*}{ Twist1 } & F: TGTCCGCGTCCCACTAGC & 60 & 93 \\
\hline & R: TGTCCATTITCTCCTTCTCTGGA & & \\
\hline \multirow[t]{2}{*}{ Aggrecan } & F: GCCTACGAAGCAGGCTATGA & 60 & 136 \\
\hline & R: GCACGCCATAGGTCCTGA & & \\
\hline \multirow[t]{2}{*}{ Type II collagen } & F: CAGGTGAACCTGGACGAGAG & 60 & 86 \\
\hline & R: ACCACGATCTCCCTTGACTC & & \\
\hline \multirow[t]{2}{*}{ RPL13A } & F: CCTGGAGGAGAAGAGGAAAGAGA & 60 & 126 \\
\hline & R: TTGAGGACCTCTGTGTATTTGTCAA & & \\
\hline
\end{tabular}

F, forward; qRT-PCR, quantitative real-time RT-PCR; $R$, reverse; RT-PCR, reverse transcriptase-polymerase chain reaction; $T_{A}$, annealing temperature.
HSG cells grown on chamber slides (Nunc, Rochester, NY, USA) were fixed in cold methanol and used for immunofluorescence staining. To unmask all antigens except type II collagen, the tissue sections were placed in a $0.01 \mathrm{M}$ citrate buffer (pH 6.0) and heated to $125^{\circ} \mathrm{C}$ for $5 \mathrm{~min}$. For type II collagen, the sections were treated with $2 \%$ bovine testicular hyaluronidase (Sigma, St Louis, MO, USA) at $37^{\circ} \mathrm{C}$ for $60 \mathrm{~min}$.

For immunoperoxidase staining, tissue sections were treated with $0.3 \% \mathrm{H}_{2} \mathrm{O}_{2}$ to block endogenous peroxidase, and then with normal goat serum (1:10) to block nonspecific binding of antibodies. Thereafter, they were incubated with primary antibodies overnight at $4{ }^{\circ} \mathrm{C}$, and then with secondary antibodies for $30 \mathrm{~min}$. Primary antibodies were rabbit antibodies to Sox6 (1:200; Sigma), Sox5 (1:100; Abcam, Cambridge, UK), and aggrecan (1:100; LifeSpan, Seattle, WA, USA); mouse antibodies to Sox9 (1:1000; Abnova, Taipei, Taiwan), Twist1 (1:1000; Abcam), and type II collagen (1:500; Daiichi Fine Chemical, Toyama, Japan). Secondary antibodies were horseradish peroxidase-conjugated polymers (EnVision+ Mouse/HRP and EnVision+ Rabbit/HRP; Dako, Carpinteria, CA, USA). Immunoreaction sites were visualized by treatment with 3,3'-diaminobenzidine tetrahydrochloride$\mathrm{H}_{2} \mathrm{O}_{2}$ solution. Finally, sections were counterstained with

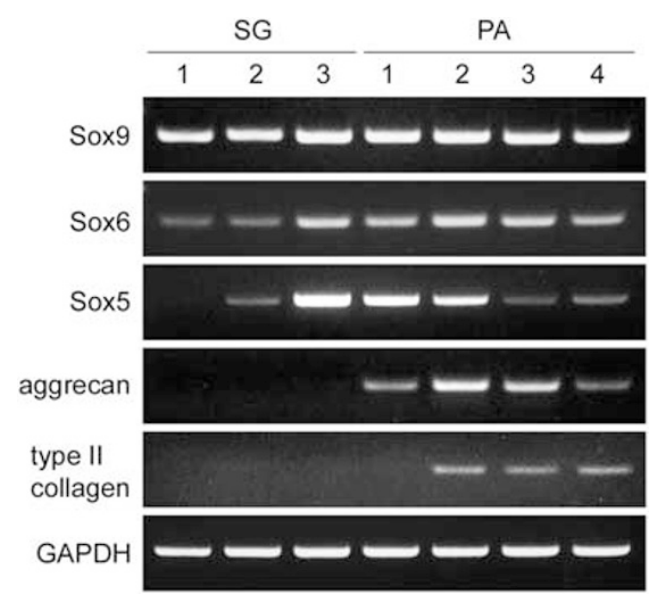

Figure 1 mRNA expression of Sox9, Sox6, Sox5, aggrecan, and type II collagen in salivary gland (SG1: palatine gland; SG2: palatine gland; SG3: submandibular gland) and pleomorphic adenoma (PA1: in palatine gland; PA2: in palatine gland; PA3: in parotid gland; PA4: in submandibular gland). Reverse transcriptase-polymerase chain reaction (RT-PCR) was used. Sox, SRY-related HMG-box.

Figure 2 Immunohistochemical expression of Sox9, Sox6, aggrecan, and type II collagen in pleomorphic adenoma (a-d and $\mathbf{g}$ ) and salivary gland $(\mathbf{e}, \mathbf{f}$, and $\mathbf{h})$. (a-f) Immunoperoxidase staining. Nuclei are stained with hematoxylin. (g and $\mathbf{h})$ Immunofluorescence staining. Nuclei are stained with DAPI (4',6-diamidino-2-phenylindole). In pleomorphic adenoma, Sox9 (a), Sox6 (b), and aggrecan (c) are seen in both epithelial and myxochondroid tissues. Type II collagen (d) is almost always seen in myxochondroid tissues and occasionally seen in epithelial tissues (upper right in d). Sox9 and Sox6 are localized in the same cells in both epithelial ( $E$ in $\mathbf{g}$ ) and myxochondroid tissues ( $M$ in $\mathbf{g}$ ). In salivary gland (submandibular gland), Sox9 (e) and Sox6 (f) are seen in duct and acinar cells. They are localized in the same cells (h). Bars: $50 \mu \mathrm{m}$ (a-f) and $25 \mu \mathrm{m}$ (g and $\mathbf{h})$. Sox, SRY-related HMG-box. 

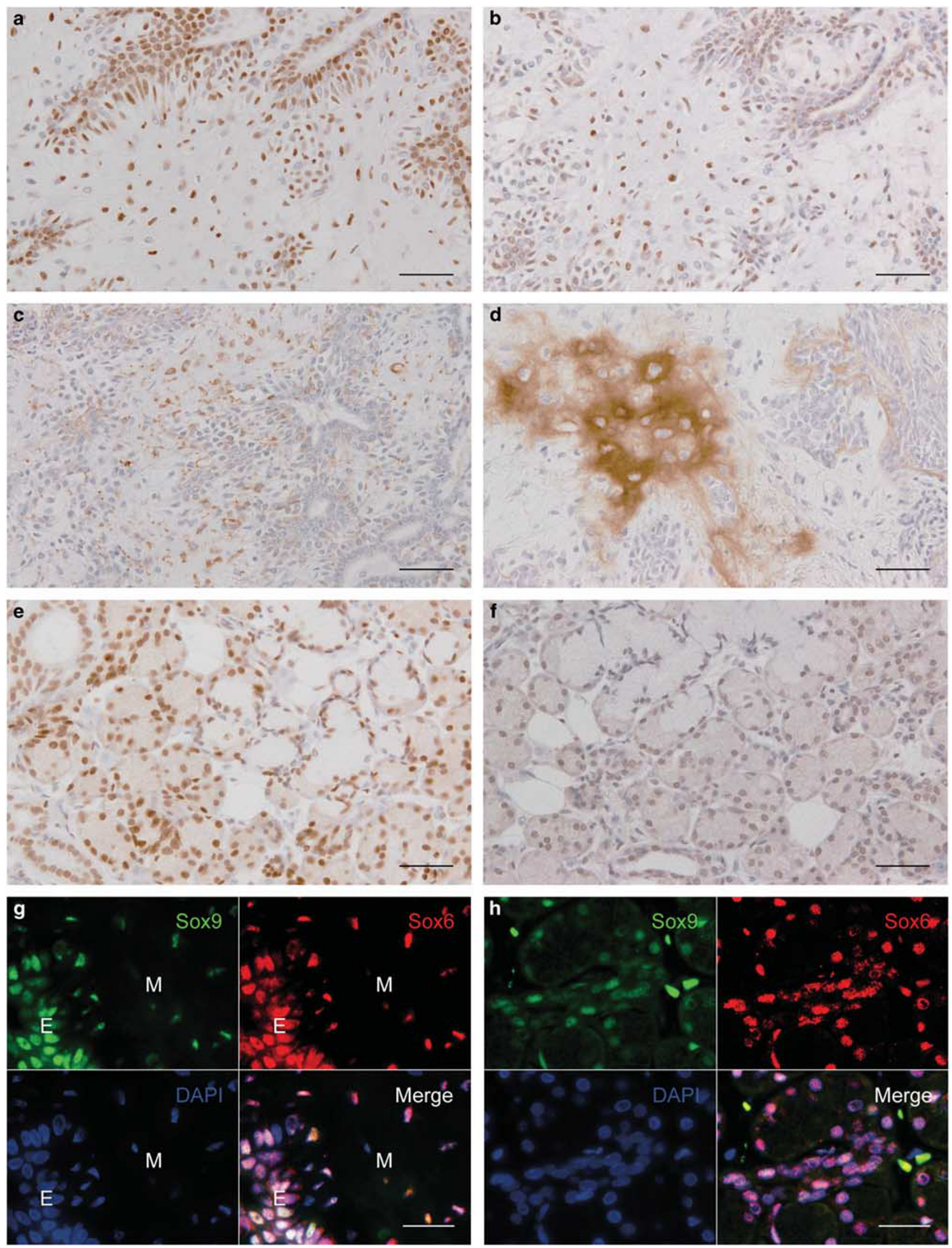
hematoxylin, coverslipped with Permount, and examined with a Nikon Eclipse E600 microscope (Nikon, Tokyo, Japan).

For immunofluorescence staining, tissue sections and cells were treated with normal donkey serum (1:10). They were then incubated with primary and secondary antibodies as described above. Primary antibodies were as follows: rabbit antibodies to Sox9 (1:200; Sigma) and Sox6 (1:200; Sigma); mouse antibodies to Sox9 (1:1000; Abnova) and Twist1 (1:1000, Abcam). Secondary antibodies, which were purchased from Jackson (West Grove, PA, USA), were FITC-conjugated donkey anti-mouse IgG (1:200), FITC-conjugated donkey anti-rabbit $\operatorname{IgG}$ (1:200), Cy3-conjugated donkey anti-mouse $\operatorname{IgG}$ (1:400), and Cy3-conjugated donkey anti-rabbit IgG (1:400). After incubation with antibodies, the sections and the cells were coverslipped with Vectashield with DAPI (Vector Laboratories, Burlingame, CA, USA) and examined with a Zeiss Axioplan fluorescence microscope (Carl Zeiss, Göttingen, Germany).

a
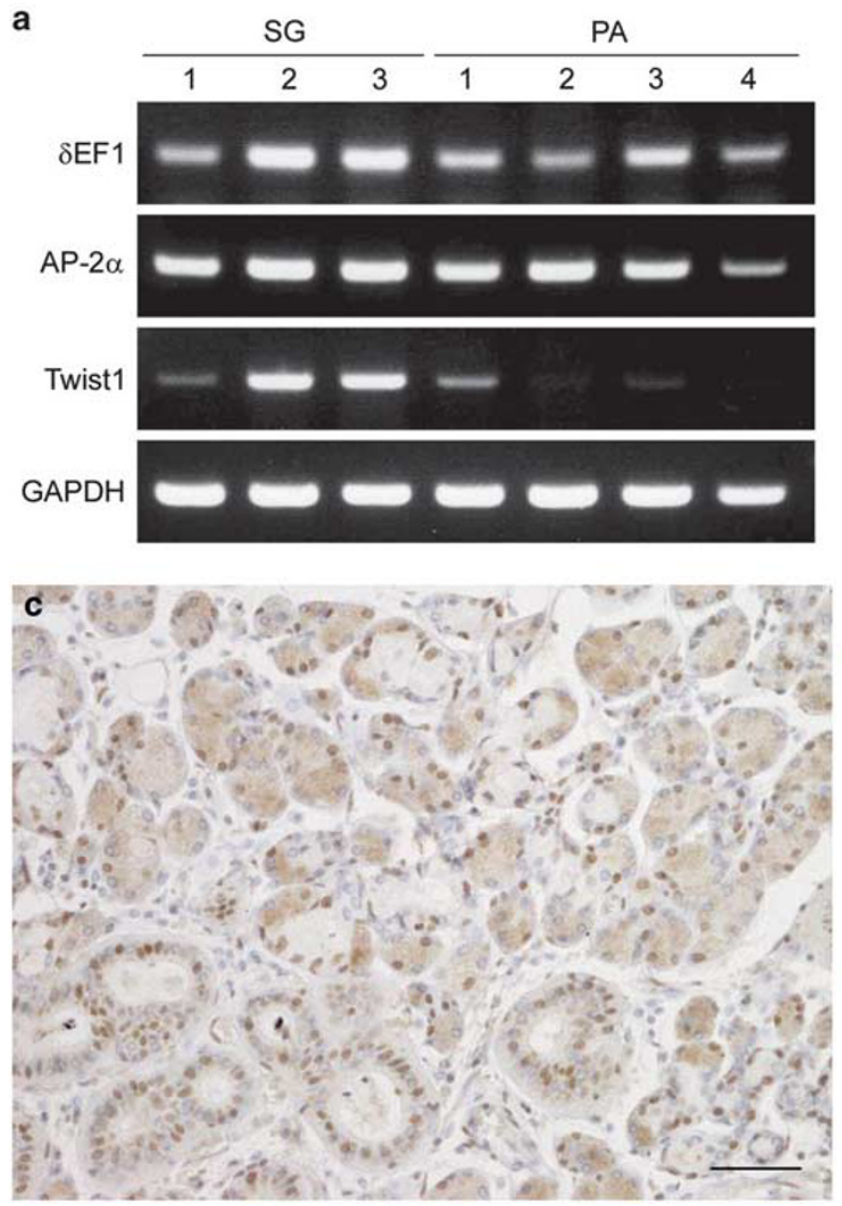

\section{Transfection with Twist1 siRNA}

HSG cells (at $>50 \%$ confluence) were transfected with $50 \mathrm{nM}$ Twist1 siRNA (Mission siRNA SASI_Hs01_00048450; Sigma) or control siRNA (SIC-001; Sigma) using Mission siRNA transfection reagent (Sigma) according to the manufacturer's instructions. After $48 \mathrm{~h}$, RNA was extracted from the cells.

\section{Transfection with Twist1 cDNA}

HSG cells (at $\sim 75 \%$ confluence) were transfected with pCMV6-Twist1 (RC202920; OriGene, Rockville, MD, USA) or pCMV6-Entry control (PS100001; OriGene) using ViaFect transfection reagent (Promega, Madison, WI, USA) according to the manufacturer's instructions. Forty-eight hours after transfection, cells were selected by growing in the medium containing $2.5 \mathrm{mg} / \mathrm{ml} \mathrm{G} 418$ (Roche Diagnostics, Indianapolis, IN, USA). The G418-resistant cell colonies were pooled, and then a mixed population of drug-resistant transfectants were grown for subsequent analysis.
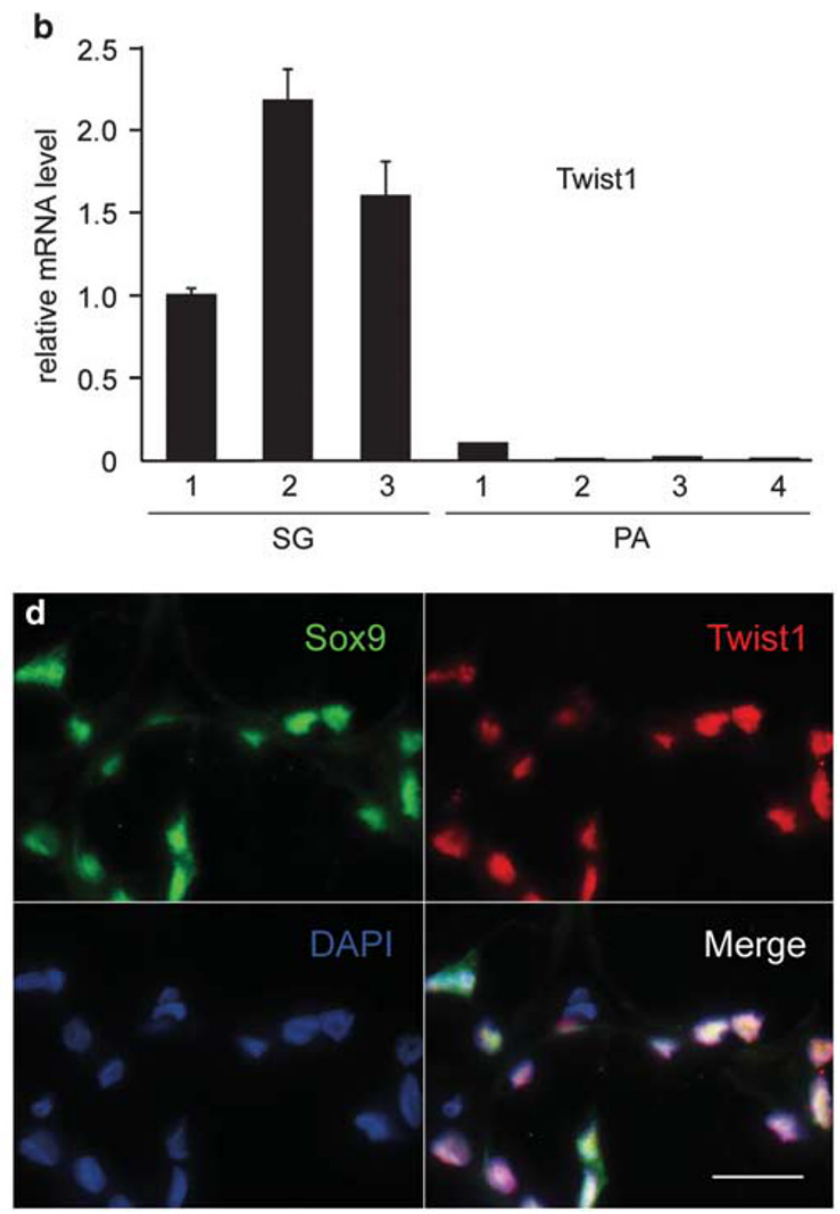

Figure 3 Expression of transcription factors that negatively regulate chondrogenesis. (a and b) Salivary gland (SG1: palatine gland; SG2: palatine gland; SG3: submandibular gland) and pleomorphic adenoma (PA1: in palatine gland; PA2: in palatine gland; PA3: in parotid gland; PA4: in submandibular gland). RT-PCR (a) and qRT-PCR (b) were used. qRT-PCR data are represented as the mean \pm s.d. of duplicate determinations. (c) Immunoperoxidase staining of Twist1 in submandibular gland. Nuclei are stained with hematoxylin. Twist1 is seen in many cells in both ducts and acini. (d) Immunofluorescence staining of palatine gland. Nuclei are stained with DAPI. Note the colocalization of Twist 1 and Sox 9 in the cells of acini. Bars: $50 \mu$ m (c) and $25 \mu \mathrm{m}$ (d). Sox, SRY-related HMG-box. 


\section{Statistical Analysis}

Statistical analysis was performed using the Student's $t$-test. Differences were considered significant when $P<0.05$.

\section{RESULTS}

mRNA Expression of Sox9, Sox6, Sox5, Aggrecan, and Type II Collagen

RNA was extracted from fresh tissues, and the expression of transcription factors positively regulating chondrogenesis was examined by reverse transcriptase-polymerase chain reaction (RT-PCR) (Figure 1). Sox9, Sox6, and Sox 5 mRNAs were detected in all four tumor tissues, Sox 9 and Sox6 mRNAs were detected in all three salivary gland tissues, and Sox 5 mRNA was detected in two gland tissues. Of the ECM substances specific to cartilage, aggrecan mRNA was detected in all tumor tissues and type II collagen mRNA was in three tumor tissues. Aggrecan and type II collagen mRNAs were not detected in any of the gland tissues.

\section{Immunohistochemical Expression of Sox9, Sox6, Aggrecan, and Type II Collagen}

Immunohistochemistry was performed on formalin-fixed paraffin-embedded tissue sections to localize the proteins of Sox9, Sox6, aggrecan, and type II collagen (Figure 2). Hematoxylin-and-eosin stained sections showed that all pleomorphic adenomas contained both epithelial and mesenchymal components. The mesenchymal component was composed of myxoid or myxochondroid tissues. Sox9 was localized in almost all the tumor cells of both the epithelial and mesenchymal components (Figure 2a). Sox6 was localized in many tumor cells of both the epithelial and mesenchymal components (Figure 2b). Sox6 was colocalized with Sox9 in these cells (Figure 2g). As with Sox9 and Sox6, aggrecan was seen in both the epithelial and mesenchymal components (Figure 2c). Type II collagen was almost always seen in the mesenchymal component and occasionally in the epithelial component (Figure 2d). In salivary glands, Sox9 was localized in almost all the acinar and duct cells (Figure 2e). Sox6 was also localized in many acinar and duct cells (Figure 2f). It was colocalized with Sox9 in these cells (Figure $2 \mathrm{~h}$ ). Sox 5 antibody did not detect Sox 5 protein either in tumor or normal tissues (data not shown).

\section{Expression of Transcription Factors that Negatively Regulate Chondrogenesis}

Expression of transcription repressors of chondrogenesis was examined in salivary glands and pleomorphic adenomas. RT-PCR detected $\delta E F 1$ and AP- $2 \alpha$ mRNAs in salivary glands and pleomorphic adenomas. Interestingly, Twistl mRNA was found in salivary glands, but was not detected in three of the four pleomorphic adenomas (PA2, PA3, and PA4 in Figure $3 \mathrm{a})$. In the remaining tumor (PA1), quantitative realtime RT-PCR (qRT-PCR) showed the mRNA level of Twist1 was significantly lower $(P<0.01)$ compared with those in salivary glands (Figure $3 \mathrm{~b}$ ). Immunohistochemistry of salivary glands showed that Twistl protein was localized in many acinar and duct cells (Figure 3c). Double-labeling immunohistochemistry showed that Twist1 was colocalized with Sox9 in the nuclei of these cells (Figure 3d).

\section{Effect of Twist1 on Chondrogenic Marker Genes Expression in HSG Cells}

The above observations prompted us to investigate whether Twistl might be involved in cartilage-like tissue formation in pleomorphic adenoma of the salivary gland. Because of technical limitations of in vitro experiments using primary salivary gland cells, we have used a human submandibular gland cell line, HSG, which are epithelial-type cells with cuboidal or somewhat elongated morphology (Figure 4b, see Shirasuna et al. ${ }^{25}$ ). Similar to the salivary gland cells described above, HSG cells expressed the mRNAs and proteins of Sox9, Sox6, and Twist1 (Figures $4 \mathrm{a}$ and b), but not those of aggrecan or type II collagen (data not shown). When HSG

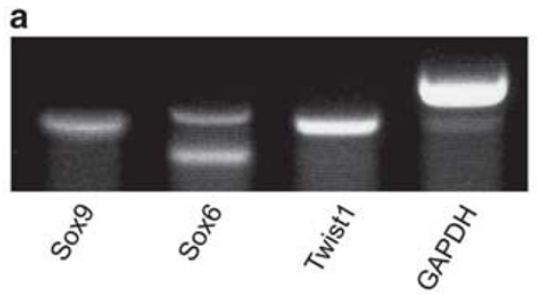

b
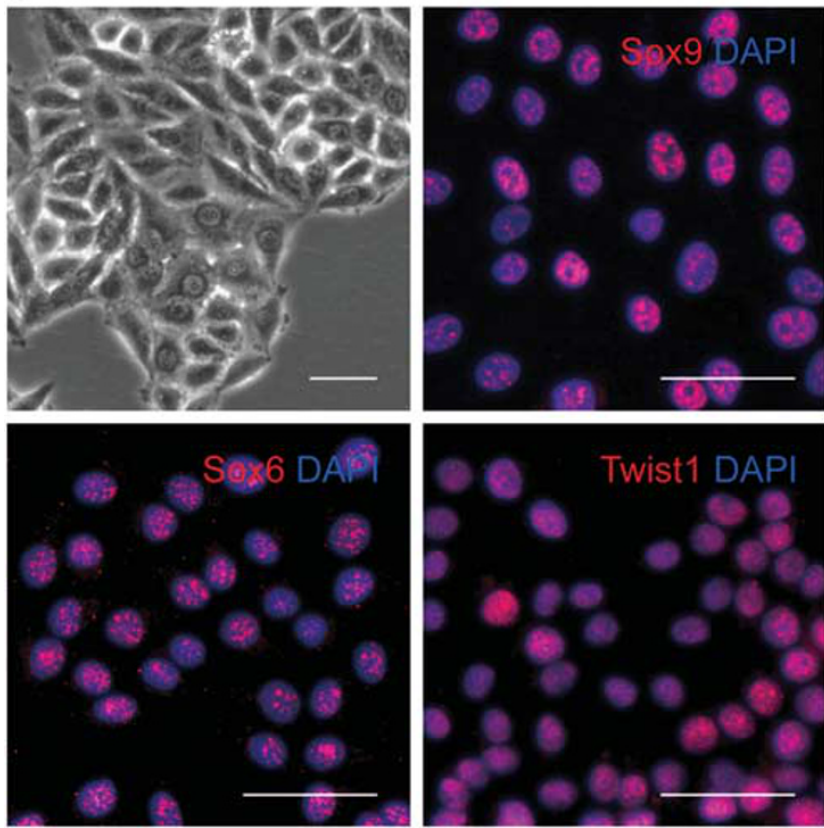

Figure 4 Expression of Sox9, Sox6, and Twist1 in human submandibular gland (HSG) cells. (a) RT-PCR was used. (b) Immunofluorescence staining. Nuclei were stained with DAPI. Note that Sox9, Sox6, and Twist1 proteins are localized in nuclei of HSG cells. A phase-contrast image of HSG cells is also shown. Bars: $25 \mu \mathrm{m}$. Sox, SRY-related HMG-box. 
cells were transfected with Twist1 siRNA, Twist1 mRNA level was reduced to $14 \%$. The mRNA levels of both aggrecan and type II collagen were significantly increased, to more than six times higher in cells transfected with Twistl siRNA compared with that in the cells transfected with control siRNA (Figure 5a). In contrast, Twist1 overexpression increased Twistl mRNA level by more than 12 times. It significantly reduced mRNA levels of both aggrecan and type II collagen, to $1 \%$ and $56 \%$, respectively, of levels seen in cells transfected with control plasmid (Figure 5b). No apparent morphological changes were observed in HSG cells after transfection with either Twist1 siRNA or Twist1 cDNA (data not shown).

\section{DISCUSSION}

This study provides indirect but conclusive evidence that epithelial cells differentiate to chondrocytes during tumorigenesis of salivary gland pleomorphic adenoma. Epithelial cells expressed Sox9 and Sox6, and produced aggrecan and type II collagen, which are ECM substances specific to cartilage. Sox9, Sox6, and Sox5 constitute a transcription factor trio essential for chondrocyte differentiation. Sox9
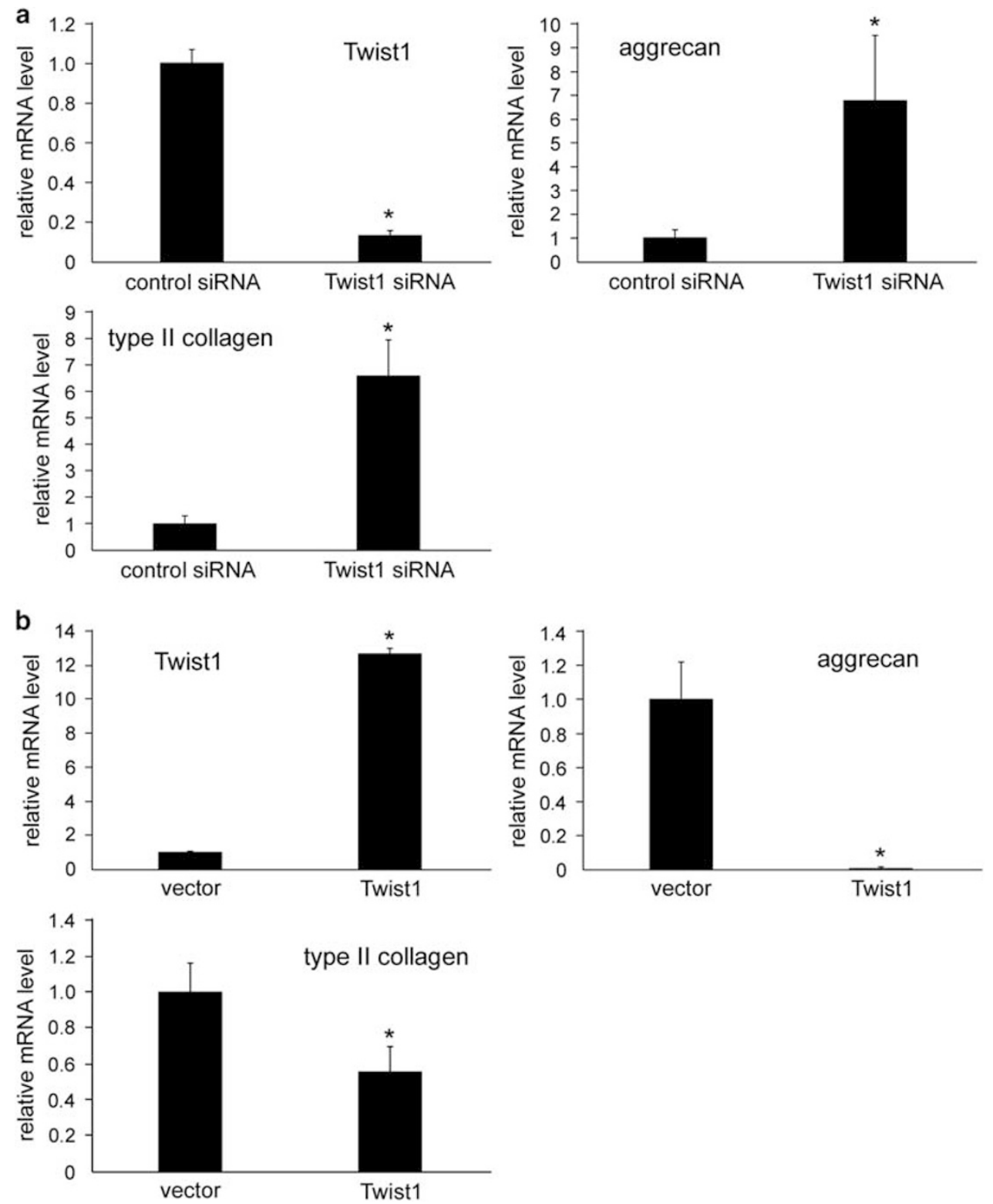

Figure 5 Effects of knockdown (a) or overexpression (b) of Twist1 gene in human submandibular gland (HSG) cells. qRT-PCR was used. Data are represented as mean \pm s.d. of duplicate determinations. For type II collagen expression in (b), determination was carried out in quadruplicate. Similar results were obtained from three independent experiments. ${ }^{*} P<0.05$ compared with control short interfering RNA (siRNA) or control plasmid vector. 
acts together with Sox6 or Sox 5 as the master regulator for chondrocyte differentiation. ${ }^{11}$ This trio of transcription factors can induce chondrocyte differentiation in both chondrogenic mesenchymal cells and nonchondrogenic cells already committed to other lineages, including epithelial cells derived from the cervix, liver, and kidney. ${ }^{10}$

Mouse salivary gland cells have been shown to express Sox $9,{ }^{24}$ as do human salivary gland cells. Both duct and acinar cells expressed Sox9. These cells were similar to pleomorphic adenoma epithelial cells in that they expressed Sox6, but were dissimilar to pleomorphic adenoma epithelial cells in that they did not produce aggrecan or type II collagen; aggrecan and type II collagen genes were not transcribed in salivary glands.

RNA transcription, the most important step in control of gene expression, depends on a balance of positive and negative transcription factors. Negative transcription factors repress positive transcription factors when and where given genes should not be expressed. For cartilage-specific genes, these include $\delta E F 1$, AP- $2 \alpha$, Snail, Slug, Twist1, and $\mathrm{C} / \mathrm{EBP} \beta .^{18-22}$ Among these, $\delta \mathrm{EF} 1, \mathrm{AP}-2 \alpha$, and Twist1 are expressed by chondrocyte progenitors and maintain their undifferentiated state. ${ }^{18,20,21,23}$ Their expression in the salivary gland was therefore compared with their expression in pleomorphic adenoma. The mRNAs of $\delta E F 1$ and AP- $2 \alpha$ were detected in both salivary glands and pleomorphic adenomas. Although the mRNA of Twistl was detected in salivary glands, Twist1 mRNA was not detected in three of the four pleomorphic adenomas. In qRT-PCR analysis, significantly less Twist1 mRNA was seen in the remaining tumor compared with that in the salivary glands. Immunohistochemistry showed that Twist1 and Sox9 proteins were localized in the same salivary gland cells. These results suggest that Twist 1 expression could repress transactivation potential of Sox proteins in salivary gland cells and that Twistl depletion, which occurs during the neoplastic transformation of salivary gland cells, permits Sox proteins to transcribe the genes of aggrecan and type II collagen.

Twist1, a member of the basic helix-loop-helix family of transcription factors, is essential for the development and specification of tissues with a mesodermal origin. Twistl has been highlighted for its function in epithelial-to-mesenchymal transition and metastasis. ${ }^{27}$ In cartilage, Twist 1 has been identified as a downstream mediator of canonical Wnt signaling, which represses chondrocyte differentiation. ${ }^{21}$ In mouse chondrocyte precursors, ectopic expression of Twist1 repressed, whereas Twistl depletion enhanced the expression of chondrocyte marker genes, including type II collagen and aggrecan. $^{21}$ Twist 1 regulates target gene expression directly or indirectly. In chondrocyte differentiation, Twist1 acts indirectly; it binds to Sox9 and inhibits Sox9's transactivation potential. ${ }^{23}$ Twist1 also binds to MyoD and Runx2, master transcription regulators of myogenesis and osteogenesis, respectively, and inhibits differentiation of mesenchymal progenitor cells toward either of these lineages. ${ }^{27}$
We hypothesized that Twist1 prevents salivary gland cells from differentiating into chondrocytes. To test this, in vitro gain- and loss-of-function experiments for Twist1 were performed in HSG cells, and expression of aggrecan and type II collagen in these cells was examined. HSG cells are immortalized cells derived from the human submandibular gland. ${ }^{26}$ Although neoplastic when inoculated into immunodeficient mice, they retain many characteristics of salivary duct cells from which pleomorphic adenoma arises. ${ }^{26}$ Our study showed that they also retain characteristics of salivary duct cells, as described above: they expressed Sox9, Sox6, and Twist1, but not aggrecan or type II collagen. Knockdown of Twist1 by siRNA resulted in the upregulation of both aggrecan and type II collagen gene expression. In contrast, Twist1 overexpression resulted in the downregulation of these two genes. These results support our hypothesis.

In conclusion, this study indicates that salivary gland cells have potential for chondrogenesis, as evidenced by expression of the Sox proteins. Our model predicts that Twist1 expression prevents salivary gland cells from fulfilling the chondrogenic potential, and Twistl depletion concomitant with neoplastic transformation permits them to differentiate toward chondrocytes and produce cartilage-like mesenchymal tissues in pleomorphic adenoma.

\section{DISCLOSURE/CONFLICT OF INTEREST}

The authors declare no conflict of interest.

1. Eveson JW, Kusafuka K, Stenman G et al. Pleomorphic adenoma. In: Barnes L, Eveson JW, Reichart P, Sidransky D (eds). WHO Classification of Tumours: Pathology and Genetics of Head and Neck Tumours. Lyon, France: IRAC Press. 2005, pp 254-258.

2. Ellis GL, Auclair PL. Tumors of the salivary gland. In: AFIP Atlas of Tumor Pathology, 4th series. Silver Spring, MD, USA: ARP Press. 2008, pp 49-71.

3. Dardick I, van Nostrand AW, Jeans MT et al. Pleomorphic adenoma, I: ultrastructural organization of 'epithelial' regions. Hum Pathol 1983;14: 780-797.

4. Dardick I, Van Nostrand AW, Jeans MT et al. Pleomorphic adenoma, II: ultrastructural organization of 'stromal' regions. Hum Pathol 1983;14: 798-809.

5. Erlandson RA, Cardon-Cardo C, Higgins PJ. Histogenesis of benign pleomorphic adenoma (mixed tumor) of the major salivary glands. An ultrastructural and immunohistochemical study. Am J Surg Pathol 1984;8:803-820.

6. Aigner T, Neureiter D, Völker $U$ et al. Epithelial-mesenchymal transdifferentiation and extracellular matrix gene expression in pleomorphic adenomas of the parotid salivary gland. J Pathol 1998;186:178-185.

7. Kusafuka K, Yamaguchi A, Kayano $\mathrm{T}$ et al. Expression of bone morphogenetic proteins in salivary pleomorphic adenomas. Virchows Arch 1998;432:247-253.

8. Zhao M, Takata T, Ogawa I et al. Immunohistochemical demonstration of bone morphogenetic protein-2 and type II collagen in pleomorphic adenoma of salivary glands. J Oral Pathol Med 1998;27:293-296.

9. Zhao M, Takata T, Ogawa I et al. Immunohistochemical evaluation of the small and large proteoglycans in pleomorphic adenoma of salivary glands. J Oral Pathol Med 1999;28:37-42.

10. Ikeda T, Kamekura S, Mabuchi A et al. The combination of SOX5, SOX6, and SOX9 (the SOX trio) provides signals sufficient for induction of permanent cartilage. Arthritis Rheum 2004;50:3561-3573.

11. Lefebvre V, Smits P. Transcriptional control of chondrocyte fate and differentiation. Birth Defects Res C 2005;75:200-212.

12. Bi W, Deng JM, Zhang Z et al. Sox 9 is required for cartilage formation. Nat Genet 1999;22:85-89. 
13. Akiyama $\mathrm{H}$, Chaboissier $\mathrm{MC}$, Martin JF et al. The transcription factor Sox9 has essential roles in successive steps of the chondrocyte differentiation pathway and is required for expression of Sox5 and Sox6. Genes Dev 2002;16:2813-2828.

14. Lefebvre V, Li P, de Crombrugghe B. A new long form of Sox5 (L-Sox5), Sox6 and Sox9 are coexpressed in chondrogenesis and cooperatively activate the type II collagen gene. EMBO J 1998;17:5718-5733.

15. Smits P, Li P, Mandel J et al. The transcription factors L-Sox 5 and Sox6 are essential for cartilage formation. Dev Cell 2001;1:277-290.

16. Han Y, Lefebvre V. L-Sox5 and Sox6 drive expression of the aggrecan gene in cartilage by securing binding of Sox9 to a far- upstream enhancer. Mol Cell Biol 2008;28:4999-5013.

17. Okazaki K, Sandell LJ. Extracellular matrix gene regulation. Clin Orthop Relat Res 2004;427:S123-S128.

18. Murray $\mathrm{D}$, Precht $\mathrm{P}$, Balakir $\mathrm{R}$ et al. The transcription factor $\delta E F 1$ is inversely expressed with type II collagen mRNA and can repress Col2al promoter activity in transfected chondrocytes. J Biol Chem 2000;275: 3610-3618.

19. Seki K, Fujimori T, Savagner $\mathrm{P}$ et al. Mouse Snail family transcription repressors regulate chondrocyte, extracellular matrix, type II collagen, and aggrecan. J Biol Chem 2003;278:41862-41870.

20. Huang $\mathrm{Z}, \mathrm{Xu} \mathrm{H}$, Sandell L. Negative regulation of chondrocyte differentiation by transcription factor AP-2a. J Bone Miner Res 2004;19:245-255.
21. Reinhold MI, Kapadia RM, Liao Z et al. The Wnt-inducible transcription factor Twist1 inhibits chondrogenesis. J Biol Chem 2006;281: 1381-1388.

22. Ushijima T, Okazaki $\mathrm{K}$, Tsushima $\mathrm{H}$ et al. CCAAT/enhancer-binding protein $\beta$ regulates the repression of type II collagen expression during the differentiation from proliferative to hypertrophic chondrocytes. J Biol Chem 2014;289:2852-2863.

23. Gu S, Boyer TG, Naski MC. Basic helix-loop-helix transcription factor Twist1 inhibits transactivator function of master chondrogenic regulator Sox9. J Biol Chem 2012;287:21082-21092.

24. Mead TJ, Wang $Q$, Bhattaram $P$ et al. A far-upstream ( $-70 \mathrm{~kb}$ ) enhancer mediates Sox9 auto-regulation in somatic tissues during development and adult regeneration. Nucleic Acids Res 2013;41:4459-4469.

25. Shirasuna K, Watatani K, Sugiyama M et al. Isolation and characterization of different clones including myoepithelial-like variants from a clonal neoplastic epithelial duct cell line of human salivary gland origin. Cancer Res 1986;46:1418-1426.

26. Shirasuna K, Sato M, Miyazaki T. A neoplastic epithelial duct cell line established from an irradiated human salivary gland. Cancer 1981;48: 745-752.

27. Qin Q, Xu Y, He T et al. Normal and disease-related biological functions of Twist1 and underlying molecular mechanisms. Cell Res 2012;22: 90-106. 